\title{
Adiabatic Invariants and Scalar Fields in a de Sitter Space-Time
}

\author{
C. Bertoni* \\ Istituto di Radioastronomia, via Gobetti 101, 40129 Bologna Italy \\ F. Finelli† G. Venturi \\ Dipartimento di Fisica, Università degli Studi di Bologna and I.N.F.N. \\ via Irnerio, 46 - 40126 Bologna - Italy
}

August 28, 2018

\begin{abstract}
The method of adiabatic invariants for time dependent Hamiltonians is applied to a massive scalar field in a de Sitter space-time. The scalar field ground state, its Fock space and coherent states are constructed and related to the particle states. Diverse quantities of physical interest are illustrated, such as particle creation and the way a classical probability distribution emerges for the system at late times.
\end{abstract}

*e-mail: bertoni@astbo1.bo.cnr.it

${ }^{\dagger} \mathrm{e}-\mathrm{mail}$ : finellif@bo.infn.it

${ }^{\ddagger} \mathrm{e}-$ mail: armitage@bo.infn.it 
In a previous paper [1] we illustrated the Born-Oppenheimer (BO) approach to the matter-gravity system in a simple minisuperspace model and in such a context the simplifications associated with the use of adiabatic invariants [2] were pointed out. Indeed through the use of such invariants one can improve on the adiabatic approximation and obtain better results for the evaluation of fluctuations which, in a quantum gravitational context, are associated with the creation of matter.

The purpose of this note is to illustrate, within the context of field theory in a curved space-time (de Sitter with flat spatial section in our simplified model), the use of adiabatic invariants with a particular emphasis on the description of the vacuum and the space of physical states. The usefulness of the method of invariants for the calculation of the geometrical (adiabatic) and dynamical phases has been previously noticed [3] and the method of invariants itself applied to quantum cosmology [4], although not in a BO context. Here we shall apply the method to the equation for matter obtained [1] in the BO approach, on neglecting fluctuations and in the semiclassical limit for gravity. This novel application allows for the time variation of the metric in a second quantized (Schrödinger functional approach [5] [6]) scalar field theory thus improving on the adiabatic approximation (static metric) and including some matter creation through the use of the vacuum and the Fock space associated with quantum adiabatic invariants.

Let us consider a Friedmann-Robertson-Walker line element:

$$
d s^{2}=-d \tau^{2}+a^{2}(\tau) g_{i j} d x^{i} d x^{j}
$$

where $g_{i j}$ is the three metric for a flat three-space. We further introduce a real massive scalar field $\Phi(\vec{x}, \tau)$ whose lagrangian density is given by:

$$
\mathcal{L}=-\frac{1}{2} g^{\mu \nu} \partial_{\mu} \Phi \partial_{\nu} \Phi-\frac{1}{2} \xi R \Phi^{2}-\frac{1}{2} \mu^{2} \Phi^{2}
$$

with $R$ the Ricci scalar and decompose the scalar field on a complete basis $u_{\mathbf{k}}$ :

$$
\Phi(\vec{x}, \tau)=\sum_{\vec{k}}\left(a_{\mathbf{k}} u_{\mathbf{k}}+a_{k}^{*} u_{\mathbf{k}}^{*}\right)
$$

with

$$
u_{k}(\vec{x}, \tau)=\frac{1}{\sqrt{V}} e^{i \vec{k} \cdot \vec{x}} \phi_{k}(\tau) \equiv \frac{1}{\sqrt{V}} e^{i \vec{k} \cdot \vec{x}} \frac{\phi_{k}^{1}+i \phi_{k}^{2}}{\sqrt{2}},
$$

where we have separated $\phi_{k}$ into its real and imaginary parts, and we consider normalization to a finite volume $\mathrm{V}$.

From the above one obtains an action: 


$$
S=\sum_{\vec{k}, i} S_{k}^{i}=\frac{1}{2} \sum_{\vec{k}} \sum_{i=1,2} \int a^{3} d \tau\left(\dot{\phi}_{k}^{i 2}-\omega_{k}^{2} \phi_{k}^{i 2}\right)
$$

where we have introduced a frequency $\omega_{k}^{2}=\frac{k^{2}}{a^{2}}+\mu^{2}+\xi R$ and by the dot we denote a derivative with respect to the time $\tau$. From Eq.(5) one sees that the modes $\vec{k}$, i are independent and may therefore be considered separately, hence we need only consider a given mode. One obtains, for a mode $\vec{k}$, i, a Hamiltonian:

$$
H_{k}^{i}=\frac{1}{2 a^{3}}\left(\pi_{k}^{i 2}+a^{6} \omega_{k}^{2} \phi_{k}^{i 2}\right)
$$

where $\pi_{\mathbf{k}}^{i}=a^{3} \dot{\phi}_{k}^{i}$ and a classical equation of motion for $\phi_{\mathbf{k}}^{i}$ :

$$
\ddot{\phi}_{k}^{i}+3 \frac{\dot{a}}{a} \dot{\phi}_{k}^{i}+\omega_{k}^{2} \phi_{\mathbf{k}}^{i}=0 .
$$

In de Sitter space $\left(a=e^{H \tau}\right.$ and $R=12 H^{2}$, where $\mathrm{H}$ is the time independent Hubble costant) this equation can be rewritten in terms of the conformal time $\eta=-\frac{1}{H} e^{-H \tau}$ $\left(a=-\frac{1}{H \eta},-\infty<\eta<0\right)$ as:

$$
\left[\frac{d^{2}}{d(k \eta)^{2}}+\frac{1}{k \eta} \frac{d}{d(k \eta)}+1-\frac{\nu^{2}}{(k \eta)^{2}}\right] a^{\frac{3}{2}} \phi_{\mathbf{k}}^{i}=0
$$

which has as solutions the Bessel functions $J_{\nu}(k|\eta|)$ and $N_{\nu}(k|\eta|)$ (or equivalently the Hankel functions $H_{\nu}^{(1)}, H_{\nu}^{(2)}$ for $u_{k}, u_{k}^{*}$ respectively) with:

$$
\nu^{2}=\frac{9}{4}-\frac{\mu^{2}+12 \xi H^{2}}{H^{2}} .
$$

Further, canonical quantization $\left(\pi_{\mathbf{k}}^{i} \rightarrow-i \hbar \frac{\partial}{\partial \phi_{\mathbf{k}}^{i}}\right)$ leads to:

$$
\hat{H}_{k}^{i}=\frac{1}{2 a^{3}}\left(-\hbar^{2} \frac{\partial^{2}}{\partial \phi_{k}^{i 2}}+a^{6} \omega_{k}^{2} \phi_{k}^{i 2}\right)
$$

which of course depends explicitly on time and may be rewritten as:

$$
\hat{H}_{k}^{i}=\hbar \omega_{k}\left(\hat{a}_{k}^{i \dagger} \hat{a}_{k}^{i}+\frac{1}{2}\right)
$$

with:

$$
\begin{gathered}
\hat{a}_{k}^{i}=\left(\frac{a^{3} \omega_{k}}{2 \hbar}\right)^{\frac{1}{2}}\left(\hat{\phi}_{\mathbf{k}}^{i}+i \frac{\hat{\pi}_{\mathbf{k}}^{i}}{a^{3} \omega_{k}}\right), \\
\hat{a}_{k}^{i \dagger}=\left(\frac{a^{3} \omega_{k}}{2 \hbar}\right)^{\frac{1}{2}}\left(\hat{\phi}_{\mathbf{k}}^{i}-i \frac{\hat{\pi}_{\mathbf{k}}^{i}}{a^{3} \omega_{k}}\right) .
\end{gathered}
$$

and $\left[\hat{a}_{k}^{i}, \hat{a}_{k^{\prime}}^{i \dagger}\right]=\delta_{k k^{\prime}}$. 
A suitable method for the study of time dependent quantum systems is that of adiabatic invariants [2]. In particular a hermitian operator $\hat{I}_{k}^{i}(\tau)$ is called an adiabatic invariant if it satisfies [2]:

$$
\frac{\partial \hat{I}_{k}^{i}(\tau)}{\partial \tau}-\frac{i}{\hbar}\left[\hat{I}_{k}^{i}(\tau), \hat{H}_{k}^{i}(\tau)\right]=0
$$

The adiabatic invariant $\hat{I}_{k}^{i}$ has real, time independent, eigenvalues and in our case, can be decomposed in terms of basic linear invariants [3]:

$$
\begin{gathered}
\hat{I}_{b, k}^{i}(\tau) \equiv e^{i \Theta_{k}(\tau)} \hat{b}_{k}^{i}(\tau) \equiv \frac{e^{i \Theta_{k}}}{\sqrt{2 \hbar}}\left[\frac{\hat{\phi}_{\mathbf{k}}^{i}}{\rho_{k}}+i\left(\rho_{k} \hat{\pi}_{\mathbf{k}}^{i}-a^{3} \dot{\rho}_{k} \hat{\phi}_{\mathbf{k}}^{i}\right)\right], \\
\hat{I}_{b, k}^{i \dagger}(\tau) \equiv e^{-i \Theta_{k}(\tau)} \hat{b}_{k}^{i \dagger}(\tau) \equiv \frac{e^{-i \Theta_{k}}}{\sqrt{2 \hbar}}\left[\frac{\hat{\phi}_{k}^{i}}{\rho_{k}}-i\left(\rho_{k} \hat{\pi}_{k}-a^{3} \dot{\rho}_{k} \hat{\phi}_{k}^{i}\right)\right],
\end{gathered}
$$

where $\rho_{k}(\tau)$ is real (see however [7]) and satisfies [2]:

$$
\ddot{\rho}_{k}+3 \frac{\dot{a}}{a} \dot{\rho}_{k}+\omega_{k}^{2} \rho_{k}=\frac{1}{a^{6} \rho_{k}^{3}}
$$

with:

$$
\Theta_{k}(\tau)=\int_{-\infty}^{\tau} \frac{d \tau^{\prime}}{a^{3}\left(\tau^{\prime}\right) \rho_{k}^{2}\left(\tau^{\prime}\right)}
$$

The quadratic, hermitian, adiabatic invariant originally introduced in [2] is therefore:

$$
\hat{I}_{k}^{i}(\tau)=\hbar\left(\hat{b}_{k}^{i \dagger} \hat{b}_{k}^{i}+\frac{1}{2}\right)=\frac{1}{2}\left[\frac{\hat{\phi}_{k}^{i 2}}{\rho_{k}^{2}}+\left(\rho_{k} \hat{\pi}_{\mathbf{k}}^{i}-a^{3} \dot{\rho}_{k} \hat{\phi}_{\mathbf{k}}^{i}\right)^{2}\right],
$$

with $\left[\hat{b}_{k}^{i}, \hat{b}_{k^{\prime}}^{i \dagger}\right]=\delta_{k k^{\prime}}$. The existence of the destruction operator $\hat{b}_{k}$ allows us to introduce a vacuum state $\chi_{0}\left(\phi_{\mathbf{k}}^{i}\right)=<\phi \mid \vec{k}, 0, \tau>_{i}$ defined by:

$$
\hat{b}_{k}^{i} \chi_{0}\left(\phi_{\mathbf{k}}^{i}\right)=\left[\frac{\hat{\phi}_{\mathbf{k}}^{i}}{\rho_{k}}+i\left(\rho_{k} \hat{\pi}_{\mathbf{k}}^{i}-a^{3} \dot{\rho}_{k} \hat{\phi}_{\mathbf{k}}^{i}\right)\right] \chi_{0}\left(\phi_{\mathbf{k}}^{i}\right)=0
$$

leading to a normalized wave function:

$$
\chi_{0}\left(\phi_{\mathbf{k}}^{i}\right)=\left(\frac{1}{\rho_{k}^{2} \hbar \pi}\right)^{\frac{1}{4}} \exp \left[-\left(\frac{1}{\rho_{k}^{2}}-i a^{3} \frac{\dot{\rho}_{k}}{\rho_{k}}\right) \frac{\phi_{k}^{i 2}}{2 \hbar}\right]
$$

since $\phi_{\mathbf{k}}^{i}, \rho_{k}$ are real. We further note that the general solution to (15) may be written in terms of two solution to (7) (say $a^{-\frac{3}{2}} J_{\nu}, a^{-\frac{3}{2}} N_{\nu}$ ) as [8]:

$$
\rho_{k}=a^{-\frac{3}{2}}\left[A J_{\nu}^{2}+B N_{\nu}^{2}+2\left(A B-\frac{\pi^{2}}{4 H^{2}}\right)^{\frac{1}{2}} J_{\nu} N_{\nu}\right]^{\frac{1}{2}},
$$


where A,B are real constants. It is clear that the vacuum state is then defined up to two real parameters (or one complex parameter) [9] [10]. Let us note that the above vacuum state is quite distinct from the harmonic oscillator one, indeed one has that:

$$
\begin{aligned}
\hat{a}_{k}^{i} & =\frac{1}{2} \hat{b}_{k}^{i}\left[\rho_{k}\left(a^{3} \omega_{k}\right)^{\frac{1}{2}}+\frac{1}{\rho_{k}\left(a^{3} \omega_{k}\right)^{\frac{1}{2}}}+i \dot{\rho}_{k}\left(\frac{a^{3}}{\omega_{k}}\right)^{\frac{1}{2}}\right]+ \\
& +\frac{1}{2} \hat{b}_{k}^{i \dagger}\left[\rho_{k}\left(a^{3} \omega_{k}\right)^{\frac{1}{2}}-\frac{1}{\rho_{k}\left(a^{3} \omega_{k}\right)^{\frac{1}{2}}}+i \dot{\rho}_{k}\left(\frac{a^{3}}{\omega_{k}}\right)^{\frac{1}{2}}\right] \equiv \\
& \equiv \mu(\tau) \hat{b}_{k}^{i}+\nu(\tau) \hat{b}_{k}^{i \dagger}
\end{aligned}
$$

and similarly for $\hat{a}_{k}^{i \dagger}$, that is the $\hat{a}_{k}^{i}$ and $\hat{b}_{k}^{i}\left(\hat{I}_{b, k}^{i}\right)$ operators are related through a Bogolubov transformation [3], and in particular one may relate them through a unitary transformation (squeezing plus rotation) [11]:

$$
\hat{a}_{k}^{i}=S(r, \varphi) R(\theta) \hat{b}_{k}^{i} R^{\dagger}(\theta) S^{\dagger}(r, \varphi)
$$

where the rotation operator $\mathrm{R}$ is:

$$
R(\theta)=\exp \left(-i \theta \hat{b}_{k}^{i \dagger} \hat{b}_{k}^{i}\right)
$$

and the squeezing operator $\mathrm{S}$ is:

$$
S(r, \varphi)=\exp \left[\frac{r}{2}\left(e^{-2 i \varphi} \hat{b}_{k}^{i 2}-e^{2 i \varphi} \hat{b}_{k}^{i \dagger 2}\right)\right]
$$

with:

$$
\left\{\begin{array}{l}
\tanh r=\left|\frac{\nu}{\mu}\right| \\
\tan \theta=\frac{I m \mu}{\operatorname{Re\mu }} \\
\tan (2 \varphi-\theta)=\frac{I m \nu}{\operatorname{Re\nu }} .
\end{array}\right.
$$

Let us remember that such a Bogolubov transformation is not unitarily implementable if one consider all modes with an infinite volume V [12]. Further we note that the above transformation is between two different Fock space basis at equal times and not between the same basis at different times.

The two different vacua will coincide in the adiabatic limit (costant frequency [2]) for which the derivatives of $\rho_{k} a^{\frac{3}{2}}$ are small. One then has from (15):

$$
\rho_{k}^{4} \simeq \frac{1}{a^{6} \omega_{k}^{2}}=\frac{1}{a^{4}}\left[k^{2}+a^{2}\left(\mu^{2}+\xi R\right)\right]^{-1}
$$

in which case $\hat{a}_{k}$ and $\hat{b}_{k}$ coincide and:

$$
\omega_{k} \hat{I}_{k}^{i}(\tau) \simeq \hat{H}_{k}^{i}(\tau)
$$


This will occur for very early times $(\tau \rightarrow-\infty)$ or for wavelengths $\frac{2 \pi a}{k}$ which are very small compared to the de Sitter horizon $H^{-1}$. In such a limit $\left(-k \eta=\frac{k}{H} e^{-H \tau} \rightarrow \infty\right)$ Eq. (20) leads to (with $\Lambda=\frac{k}{H} e^{-H \tau}-\frac{\pi}{2} \nu-\frac{\pi}{4}$ ):

$$
\frac{1}{k}=\frac{2 H}{\pi k}\left(A \cos ^{2} \Lambda+B \sin ^{2} \Lambda+2\left(A B-\frac{\pi^{2}}{4 H^{2}}\right)^{\frac{1}{2}} \sin \Lambda \cos \Lambda\right),
$$

which has as solution:

$$
\begin{gathered}
A=B=\frac{\pi}{2 H} \\
\rho_{o k}=a^{-\frac{3}{2}}\left(\frac{\pi}{2 H}\right)^{\frac{1}{2}}\left(J_{\nu}^{2}+N_{\nu}^{2}\right)^{\frac{1}{2}}
\end{gathered}
$$

whereupon eq. (19) becomes:

$$
\chi_{0}\left(\phi_{\mathbf{k}}^{i}\right)=\left(\frac{1}{\rho_{0 k}^{2} \hbar \pi}\right)^{\frac{1}{4}} \exp \left[-\left(\frac{1}{\rho_{0 k}^{2}}-i a^{3} \frac{\dot{\rho}_{0 k}}{\rho_{0 k}}\right) \frac{\phi_{k}^{i 2}}{2 \hbar}\right] .
$$

The expressions we have obtained for the vacuum in Eqs.(19), (30), although apparently different, are actually the same as those previously obtained [10]. Indeed one may write:

$$
\frac{1}{\rho_{k}^{2}}-i a^{3} \frac{\dot{\rho_{k}}}{\rho_{k}}=-i a^{3} \frac{\dot{\psi_{k}}}{\psi_{k}}+\frac{3}{2} i a^{2} \dot{a}
$$

where:

$$
\frac{\psi_{k}^{\prime}}{\psi_{k}}=\frac{\frac{1}{2} \frac{d}{d \tau}\left\{\left[J_{\nu}+\frac{1}{A}\left(A B-\frac{\pi^{2}}{4 H^{2}}\right)^{\frac{1}{2}} N_{\nu}\right]^{2}+\frac{N_{\nu}^{2} \pi^{2}}{4 A^{2} H^{2}}\right\}+\frac{i}{A}}{\left[J_{\nu}+\frac{1}{A}\left(A B-\frac{\pi^{2}}{4 H^{2}}\right)^{\frac{1}{2}} N_{\nu}\right]^{2}+\frac{N_{\nu}^{2} \pi^{2}}{4 A^{2} H^{2}}}
$$

which for the solution Eq. (29) becomes:

$$
\frac{\dot{\psi}_{k}}{\psi_{k}}=\frac{\dot{J}_{\nu} J_{\nu}+\dot{N}_{\nu} N_{\nu}+\frac{2 i H}{\pi}}{J_{\nu}^{2}+N_{\nu}^{2}}=\frac{\dot{J}_{\nu}-i \dot{N}_{\nu}}{J_{\nu}-i N_{\nu}}=\frac{\dot{H}_{\nu}^{(2)}}{H_{\nu}^{(2)}} .
$$

The above result actually corresponds to the usual Bunch-Davies [9] vacuum in the Schrödinger picture [10] and we have chosen it among the diverse de Sitter invariant vacua by implementing adiabaticity for very early times.

The general vacuum state (18) which satisfies the Schrödinger equation associated with $H_{k}^{i}$ may be written as [2] [3]:

$$
\left|\vec{k}, 0, \tau>_{i, s}=e^{-i \frac{\Theta_{k}}{2}}\right| \vec{k}, 0, \tau>_{i}
$$

and we may construct arbitrary eigenstates $\mid \vec{k}, n, \tau>_{i, s}$ :

$$
|\vec{k}, n, \tau\rangle_{i, s}=e^{-i n \Theta_{k}(\tau)} \frac{\left(\hat{b}^{i \dagger}\right)^{n}}{\sqrt{n !}}|\vec{k}, 0, \tau\rangle_{i, s}
$$


and a coherent state [13] [14]:

$$
|\vec{k}, \alpha, \tau\rangle_{i, s}=e^{-|\alpha|^{2} / 2} \sum_{n=0}^{\infty} \frac{\alpha^{n}}{\sqrt{n !}}|\vec{k}, n, \tau\rangle_{i, s}
$$

where $\alpha=u+i v$ is an arbitrary constant, both of which again satisfy the Schrödinger equation associated with $\hat{H}_{k}^{i}(10)$. Let us emphasize that our construction of the above Fock space and the related coherent states through the use of adiabatic invariants generalises previous work in which only the vacuum state was analyzed [5] [10].

Given the coherent state one may evaluate (with $\left\langle O>_{s} \equiv{ }_{i, s}\langle\vec{k}, \alpha, \tau|\hat{O}| \vec{k}, \alpha, \tau\rangle_{i, s}\right.$ ):

$$
<\phi_{\mathbf{k}}^{i}>_{s}=\sqrt{2 \hbar}|\alpha| \rho_{k} \cos \left(\Theta_{k}-\delta\right)
$$

with $\tan \delta=\frac{v}{u}$,

$$
\begin{gathered}
<\pi_{\mathbf{k}}^{i}>_{s}=\sqrt{2 \hbar}|\alpha|\left[a^{3} \dot{\rho}_{k} \cos \left(\Theta_{k}-\delta\right)-\frac{1}{\rho_{k}} \sin \left(\Theta_{k}-\delta\right)\right] \\
<\left(\Delta \phi_{\mathbf{k}}^{i}\right)^{2}>_{s} \equiv<\left(\hat{\phi}_{\mathbf{k}}^{i}-<\phi_{\mathbf{k}}^{i}>_{s}\right)^{2}>_{s}=\frac{\hbar}{2} \rho_{k}^{2} \\
<\left(\Delta \pi_{\mathbf{k}}^{i}\right)^{2}>_{s} \equiv<\left(\hat{\pi}_{\mathbf{k}}^{i}-<\pi_{\mathbf{k}}^{i}>_{s}\right)^{2}>_{s}=\frac{\hbar}{2}\left(\frac{1}{\rho_{k}^{2}}+a^{6} \dot{\rho}_{k}^{2}\right),
\end{gathered}
$$

where $\left\langle\phi_{\mathbf{k}}^{i}\right\rangle_{s}$ can be verified to be a solution of eq.(7). Thus through the use of adiabatic invariants we obtain solutions to the classical equations of motion and therefore a correct definitions of physical states [15], which are related to the particle states (associated with $\left.\hat{a}, \hat{a}^{\dagger}\right)$ through a Bogolubov transformation.

Further from the above one obtains:

$$
\begin{gathered}
<\hat{I}_{k}^{i}>_{s}=\hbar\left(|\alpha|^{2}+\frac{1}{2}\right) \\
<\left(\Delta \phi_{\mathbf{k}}^{i}\right)^{2}>_{s}^{\frac{1}{2}}<\left(\Delta \pi_{\mathbf{k}}^{i}\right)^{2}>_{s}^{\frac{1}{2}}=\frac{\hbar}{2} \sqrt{1+a^{6} \rho_{k}^{2} \dot{\rho}_{k}^{2}}, \\
<H_{k}^{i}>_{s}=\frac{1}{2 a^{3}}\left[<\hat{\pi}_{k}^{i 2}>_{s}+a^{6} \omega_{k}^{2}<\hat{\phi}_{k}^{i 2}>_{s}\right] \\
=\frac{1}{2 a^{3}}\left[\frac{\hbar}{2}\left(\dot{\rho}_{k}^{2} a^{6}+\frac{1}{\rho_{k}^{2}}+a^{6} \omega_{k}^{2} \rho_{k}^{2}\right)+<\hat{\pi}_{\mathbf{k}}^{i}>_{s}^{2}+a^{6} \omega_{k}^{2}<\hat{\phi}_{\mathbf{k}}^{i}>_{s}^{2}\right] .
\end{gathered}
$$

The adiabatic limit $(\tau \rightarrow-\infty)$ for which $\rho_{k}$ is given by $1 / a k^{\frac{1}{2}}$ is immediately obtained for Eqs.(41), (42) and (43) and in particular we note that the uncertainty relation (42) is then minimal and the Fock spaces associated with $a$ and $b$ coincide (see also Eq.(27)). On the other hand on considering $\tau \rightarrow \infty$ one obtains: 


$$
\begin{gathered}
<\phi_{\mathbf{k}}^{i}>_{s} \simeq(2 \hbar B)^{\frac{1}{2}}|\alpha| a^{\nu-\frac{3}{2}}\left(\frac{k}{2 H}\right)^{-\nu} \frac{\Gamma(\nu)}{\pi} \cos \left(\Theta_{k}-\delta\right) \\
<\pi_{\mathbf{k}}^{i}>_{s} \simeq(2 \hbar B)^{\frac{1}{2}}|\alpha| H a^{\nu+\frac{3}{2}}\left(\nu-\frac{3}{2}\right)\left(\frac{k}{2 H}\right)^{-\nu} \frac{\Gamma(\nu)}{\pi} \cos \left(\Theta_{k}-\delta\right) \\
<\phi_{k}^{i 2}>_{s} \simeq \hbar B a^{2 \nu-3}\left(\frac{k}{2 H}\right)^{-2 \nu}\left(\frac{\Gamma(\nu)}{\pi}\right)^{2}\left(\frac{1}{2}+2|\alpha|^{2} \cos ^{2}\left(\Theta_{k}-\delta\right)\right) \\
<\pi_{k}^{i 2}>_{s} \simeq \hbar B H^{2}\left(\nu-\frac{3}{2}\right)^{2} a^{2 \nu+3}\left(\frac{k}{2 H}\right)^{-2 \nu}\left(\frac{\Gamma(\nu)}{\pi}\right)^{2}\left(\frac{1}{2}+2|\alpha|^{2} \cos ^{2}\left(\Theta_{k}-\delta\right)\right) \\
<\left(\Delta \pi_{k}\right)^{2}>_{s}^{\frac{1}{2}}<\left(\Delta \phi_{k}\right)^{2}>_{s}^{\frac{1}{2}} \simeq \frac{\hbar}{2} B H\left(\nu-\frac{3}{2}\right)^{2 \nu}\left(\frac{k}{2 H}\right)^{-2 \nu}\left(\frac{\Gamma(\nu)}{\pi}\right)^{2} \\
<H_{k}>_{s} \simeq \frac{\hbar}{4} B\left(\frac{\Gamma(\nu)}{\pi}\right)^{2}\left(\frac{k}{2 H}\right)^{-2 \nu} a^{2 \nu}\left[\tilde{\mu}^{2}+H^{2}\left(\nu-\frac{3}{2}\right)^{2}\right]+ \\
+\hbar|\alpha|^{2} B\left(\frac{\Gamma(\nu)}{\pi}\right)^{2} a^{2 \nu}\left(\frac{k}{2 H}\right)^{-2 \nu} \cos ^{2}\left(\Theta_{k}-\delta\right)\left[\left(\nu-\frac{3}{2}\right)^{2} H^{2}+\tilde{\mu}^{2}\right],
\end{gathered}
$$

with $\tilde{\mu}^{2}=\mu^{2}+12 \xi H^{2}$ and in the above the ground state results can be obtained by setting $\alpha=0$. It is immediate to see that $\left(<\phi_{k}^{i 2}>_{s}\right)^{\frac{1}{2}}$ with either $\alpha=0$ or $\alpha \neq 0$, that is for the ground state or an arbitrary (classical) state, has the same behaviour for $\tau \rightarrow \infty$ as $\left\langle\phi_{k}^{i}\right\rangle_{s}$ for $\alpha \neq 0$. It is for this reason that it is stated that the scalar field ground state leads to a classical probability distribution at late times [10]. Further the behaviour of $\left\langle\hat{H}_{k}\right\rangle_{s}$ at late times corresponds to particle creation (which can actually oscillates bacause of the $\cos ^{2}\left(\Theta_{k}-\delta\right)$ term [16]), this is also the reason for the increase of the uncertainties in the same limit.

Let us add a comment on the possible use of a rescaled matter field [18] $\zeta_{k}^{i}=\phi_{k}^{i} / a$ in Eq. (5). This will lead to a modified action:

$$
S=\sum_{\vec{k}, i} S_{k}^{i}=\frac{1}{2} \sum_{\vec{k}} \sum_{i=1,2} \int a d \tau\left[\dot{\zeta}_{k}^{i 2}-\Omega_{k}^{2} \zeta_{k}^{i 2}-\frac{d}{a d \tau}\left(\dot{a} \zeta_{k}^{i 2}\right)\right]
$$

where $\Omega_{k}^{2}=\frac{k^{2}}{a^{2}}+\mu^{2}+\left(\xi-\frac{1}{6}\right) R$. The solution to the classical equations of motion in Eq.(7) will be unchanged however there will be modifications in the subsequent Hamiltonian formulation, although the general structure will remain unaltered. Thus, for example, the asymptotic behaviour in Eq.(49) will remain the same, but the coefficient multiplying it changes. The presence of the boundary term in Eq.(50) is important: indeed if it is omitted (which is actually not possible with our classical solutions for $\zeta$ ) the asymptotic behaviour in Eq.(49) is again the same, but coefficient multiplying it vanishes for $\mu=0, \xi=\frac{1}{6}$ (conformal matter) corresponding to no particle creation. 
In the above we have illustrated the construction of the vacuum and physical states for a massive scalar field in a de Sitter space-time through the use of quantum adiabatic invariants and the associated coherent states. Such coherent states (in the $b$ modes) correspond to squeezed particle states (a modes), this is related to particle creation and is an improvement on the adiabatic approximation in which the classical limit is obtained

for coherent states in the particle modes $a$ and there is no particle creation [17]. Thus in a functional Schrödinger approach we have obtained not only the previously suggested vacuum [9] [10] but since we also have the Fock space we are able to include particle creation in the classical limit through the increase in the classical field amplitude.

\section{References}

[1] C. Bertoni, F. Finelli and G. Venturi, Class. Quantum Grav. 13 (1996) 2375

[2] H. R. Lewis jr. and W. B. Riesenfeld, Journal of Math. Phys. 10 (1969) 1458

[3] X.-C. Gao, J.-B. Xu and T.-Z. Quian Phys. Rev. D 44 (1991) 7016; X.-C. Gao, J.-B. $\mathrm{Xu}$ and T.-Z. Quian Ann. Phys. (N.Y.) 204 (1990) 235

[4] S. Abe, Phys. Rev. D 47 (1993) 718

[5] J. Guven, B. Lieberman and C. T. Hill, Phys. Rev. D 39 (1989) 438

[6] X.-C. Gao, J. Gao, T.-Z. Quian and J.-B. Xu, Phys. Rev. D 53 (1996) 4374

[7] L. S. Wollenberg, Phys. Lett. A 79 (1980) 269

[8] G. E. Prince and L. J. Eliezier J. Phys A 13 (1980) 815; Lutzky M 1978 Phys. Lett. A 68 (1978) 3

[9] T. S. Bunch and P. C. W. Davies, Proc. R. Soc. London A360 (1978) 117

[10] A. Guth and S.-Y. Pi, Phys. Rev. D 32 (1985) 1899

[11] B. L. Schumaker, Phys. Rep. 135 (1986) 317; L. P. Grishchuck and Y. V. Sidorov, Phys. Rev. D 42 (1990) 3413

[12] S. Kamefuchi and H. Umezawa, Nuovo Cimento 31 (1964) 429

[13] J. G. Hartley and J. R. Ray, Phys. Rev. D 25 (1982) 382

[14] J. A. Pedrosa, Phys. Rev. D 36 (1987) 1279 
[15] S. A. Fulling, Aspects of Quantum Field Theory in Curved Space-Time (Cambridge Univ. Press, 1989)

[16] B. L. Hu, G. Kang and A. Matacz, Int. J. of Mod. Phys. A 9 (1994) 991

[17] R. Casadio and G. Venturi, Class. Quantum Grav. 13 (1996) 2715

[18] N. D. Birrell and P. C. W. Davies, Quantum Fields in Curved Space (Cambridge Univ. Press, 1982) 